Proceedings of the 2011 Winter Simulation Conference

S. Jain, R.R. Creasey, J. Himmelspach, K.P. White, and M. Fu, eds.

\title{
WHY DOESN'T HEALTHCARE EMBRACE SIMULATION AND MODELING? WHAT WOULD IT TAKE?
}

\author{
James Fackler \\ The Johns Hopkins University \\ School of Medicine \\ 600 North Wolfe Street \\ Baltimore, MD 21287, USA
}

\author{
Michael Spaeder \\ The George Washington University \\ School of Medicine \\ 111 Michigan Avenue, NW \\ Washington, DC 20010, USA
}

\begin{abstract}
Physicians do modeling - every day, all day. It's just that it's done with hideous imprecision making cross-patient conclusions hazardous and extensibility impossible. Most of these mental models are devoid of formal logic. Rather, these mental models are patterns matched in a specific patient with a specific problem(s) based on a clinician's experience and "book-knowledge". We will explore some of the steps that could contribute to the broader acceptance of mathematical models in health care. We will distinguish models that impact the care of the individual patient from that of a larger population.
\end{abstract}

\section{INTRODUCTION}

Physicians do modeling - every day, all day. It's just that it's done with hideous imprecision making cross-patient conclusions hazardous and extensibility impossible.

A recent study of physician decision making within two academic critical care units discovered that during organized patient discussions physicians perform cognitive tasks within five broad categories; most relevant here is the identification of patterns to support complete, or most often fragmentary, mental models (Fackler et al 2009). Fragmentary mental models are coalesced on-the-fly as situations warrant into comprehensive just-in-time mental models that should be flexible, dynamic, and context-specific (Sieck et al 2004).

Unfortunately, most of these mental models are devoid of formal logic. Rather, these mental models are patterns matched in a specific patient with a specific problem(s) based on a clinician's experience and "book-knowledge". The more experienced clinician is, generally, a better modeler.

Yet, these models share many characteristics of "real" models. They are representations of reality. The model is used to predict responses to inputs. For example, a patient with fever, cough, productive sputum and rales should coalesce into the mental model of pneumonia. If oxygen is used as an input the response should be higher oxygen saturation in the arterial blood. If routine antibiotics do not produce substantial resolution in three to five days, the model may require adjustment.

Thus to dispense with the primary question in the title, health care has embraced modeling; healthcare just doesn't know it. The remainder of the discussion will be focused on how to improve on the current state of hideous imprecision and extensibility.

Again, models are merely a simplified representation of reality that captures some of that reality's essential properties and relationships (Stahl 2008). Mathematical models use mathematics to express reality. Practically speaking and as mentioned above, models should allow clinicians to better understand the responses to inputs into a particular system that should aid in decision analysis. 


\section{Fackler and Spaeder}

We will explore some of the steps that could contribute to the broader acceptance of mathematical models in health care. We will distinguish models that impact the care of the individual patient from that of a larger population.

\section{BREAK THE MODELED REALITY INTO SMALL PIECES}

At first blush, much of what makes modeling so attractive as a decision analysis tool in patient care is exactly what scares so many physicians. The concept that mathematics can be employed to create a modeled reality is difficult for the typical physician to comprehend. Physicians are trained largely in the biological sciences from which most mathematics is removed. The first step in demystifying models in the eyes of physicians may be to simply break down the modeled reality into small pieces and generating comfort in "super-human" solutions to "simple" problems.

Physicians definitely have at least an elementary understanding of the many feedback systems that exist in the human body (e.g. hypothalamic-pituitary-adrenal axis). While these systems can be quite complex, at their basic level, they are simply systems that consist of multiple interrelated variables. Medical educators have long relied on the method of breaking down these systems into their individual components in order to teach students how these components relate to one another. So the student learning about the body's stress response starts with the production of cortisol in the adrenal gland, which in turn, inhibits the hypothalamus and pituitary gland downstream. This approach allows the students to develop an appreciation for both the normal and abnormal function of this system.

This approach also works at the bedside. The infant with temperature instability is placed in an incubator in the nursery. How does the incubator "know" that it is providing a sufficient amount of heat to normalize the infant's temperature while not overheating the baby? The incubator simply uses a closed loop system via a transcutaneous monitor to sense the infant's body temperature (input) to regulate the ambient temperature of the incubator (output). Experienced clinicians learn to understand the child's physiology not by analyzing the input but rather by analyzing the variations in output. When the clinician begins to understand the models as extensions of the simplest components, inputs and outputs, it is monumentally easier to understand and hopefully possible to extend.

\section{CHOOSE THE RIGHT FIELD}

It can be argued that applications of modeling will generally be applicable for a broad array of medical specialties and subspecialties, we will focus on the fields of anesthesiology and intensive care medicine. Anesthesiology and intensive care medicine are not only the chosen fields of the authors, but also represent two of the fields that are most ripe for acceptance of modeling.

Anesthesiologists and intensivists have long been among the early adaptors of advanced technology in healthcare. There are descriptions of closed-loop (model-based) systems for control of anesthesia from the 1950's (Kiersey et al 1954) and blood pressure control from the 1980's (de Asla et al 1984). Just like the audiophile who requires expensive state of the art equipment with which to listen to his favorite music, anesthesiologists and intensivists often seek out the latest and greatest tools to monitor a patient's clinical status through both invasive and noninvasive means. The resulting increase in data quality, quantity and availability furnished to the physician can potentially strengthen clinical decision-making but not without the potential for data overload.

No two fields rely more heavily on physiologic data on a minute-to-minute basis than anesthesia and intensive care medicine. In the operating room and ICU, both reliable assessments of physiologic state and the responses to interventions are important for decision making. The volume of information available to the physician in the care of a critically ill patient can often be overwhelming leading to a reliance on experience and subjective decision-making. For example, on a typical morning rounds in an ICU, an intensivist can be confronted with more than 200 variables in a critically ill patient (Fried 2001), rendering it impossible for even an experienced physician to develop a mental response to any problem involv-

ing more than two or three dichotomous decisions (Miller 1956). In view of the high dimension of data 


\section{Fackler and Spaeder}

required to be analyzed in short spans of time, significant problems arise from the natural limitations of human beings (Imhoff 2002).

Within the multitude of variables analyzed in the care of the critically ill patient, multiple relationships exist. The relationships can be both direct and indirect (i.e., they are induced by other variables). Distinguishing between direct and indirect relationships among observed variables and primary and secondary consequences of medical interventions is difficult from experience alone (Imhoff 2002). The establishment of these relationships without introducing excessive noise into the system becomes an essential, albeit difficult, task for the clinician to undertake.

\section{THEY REALLY COULD USE HELP WITH REPETITIVE, EASILY MODELED TASKS}

Again, an intensive care physician may have literally hundreds of variables to consider in the care of a typical critically ill patient. While the applications of modeling have become increasingly complex in recent years, physicians can really use help with repetitive, easily modeled tasks. It follows that if the physician understands the role of modeling in the simple tasks, embracing modeling in general, will be easier.

There is a wealth of evidence that good glycemic control in critically ill patients improves morbidity and mortality outcomes (Krinsley 2008; Kavanagh 2010). The typical process involves the frequent monitoring of a patient's serum glucose level and the addition or modification of insulin therapy based on the serum glucose level and physician judgment. In recent years, there has been widespread adoption of treatment protocols related to glycemic control in an effort to reduce the variability of care and improve outcomes in critically ill patients (Chase et al 2011). Protocolized care, however, does not address the variability of outcome due to inter- and intra-patient variability, as it relates to both physiologic state and therapeutic response (Chase et al 2011). More recently, because of human fallibility and the fear of "overshoot" resulting in serious morbidity from unintended hypoglycemia, the push has not been for better models. Rather, most clinicians have shifted the target glucoses higher. It is here where the "superhuman" models would be the right place to start.

Glycemic control is one of the simple tasks in the care of the critically ill patient for which physiologic modeling has been applied. In recent years, there have been a number of papers published on this topic with a variety of modeling techniques proposed. Many authors have taken great steps to frame the debate around the use of physiologic modeling as a method to improve patient safety (Chase et al 2011). The value of models as a patient safety tool can't be underestimated, especially as it relates to increasing physician acceptance of the models.

We all know the child who refuses to eat eggs, yet will eagerly consume large quantities of chocolate chip cookies despite the fact that eggs are a major ingredient of this delicious treat. Physicians can behave in a very similar manner, expressing an outright aversion to modeling while not realizing the degree to which it is already prevalent in the management of patients. Take for example the use of ventilators to support the respiratory function of patients. In a simple mode like pressure support ventilation, flow (output) from the ventilator is constantly changing to maintain pressure (input) constant through inspiration (Branson 2002). The ventilator is using modeling, in this case a closed-loop control system, to adapt to changes in the patient's pulmonary physiology (e.g. compliance, respiratory rate).

\section{TEACH MORE MATHEMATICS IN SCHOOL}

It is a fact of life that a true appreciation of the application of models in the delivery of care requires at least some basic understanding of the mathematics involved. It is unreasonable to think that a typical medical student will receive instruction in probability theory and statistics such that they define a Markov process or construct an ARIMA model. It is reasonable, however, to advocate for increased exposure of students and trainees to mathematics and statistics as part of the medical education process.

In the United States, there is no uniform requirement regarding prerequisites in mathematics or statistics for admission to medical school as there are for the physical and biologic sciences. Some schools require one to two semesters of "college mathematics" while others encourage, but do not require, appli- 


\section{Fackler and Spaeder}

cants to undertake in coursework in calculus and statistics. In medical school, a minimal amount of biostatistics is offered and often doesn't extend beyond the instruction of the concepts of sensitivity and specificity.

In recent years, there has been an increased emphasis placed on testing biostatistical concepts on the specialty and subspecialty board exams in the United States. This increased emphasis has resulted in the improvement of postgraduate curriculums to include some instruction in statistics and biostatistics. While most curriculums "teach to the test", this is a move in the right direction. We would advocate for even more curricular improvements at both the medical school and postgraduate levels to include topics like regression analysis and data analysis.

\section{HIDE THE MATH OR CREATE ABSTRACTIONS OF THE MATH THAT MORTALS CAN UNDERSTAND.}

Of all the suggestions, this we believe to be the most important. All effective models will be automated. Transparency will be crucial. The user interface for even a "simple" glucose control model must show previous inputs (i.e., glucose), outputs (i.e., insulin doses), trends and their correlation with the expected, and a projection into the near future. The math must be reduced and/or translated into language the user can understand and trust. Where explicit algorithms cannot be articulated (as in some neural networks), approximations must be created. Recently published statements like, “...However, the Archimedes Model is large and complex and uses mathematics that may be unfamiliar to some persons... (Eddy et al 2011)" must be obliterated.

\section{BUILD INCREASINGLY COMPLEX MODELS - PATIENT SPECIFIC?}

The path to broader physician acceptance of modeling in the process of patient care delivery is impacted by a variety of issues. With greater insight, awareness and education hopefully follows broader acceptance. What may be lost in the acceptance process by the physician is the high level of innovation required for model development. Once broader acceptance has been achieved, innovation must take center stage.

Up to now, we've focused on the use of modeling to help the physician in the delivery of care of the individual patient. Healthcare modeling for the most part has focused on populations, both small and large. Infectious disease surveillance, resource allocation and optimization, health technology assessment and health policy decision-making are just a few of the applications of population level models (Burke 2006).

There has been widespread acceptance of population-based models by a variety of stakeholders health economists, health policy makers, health insurers and the pharmaceutical industry, to name a few. Governmental entities of the United States, Canada and the UK all use some form of modeling in the comparative effectiveness analysis of healthcare policies and interventions.

So where does this leave the individual clinician? In many respects, the process of acceptance of modeling at the population level is the same for acceptance of any new therapy or process in healthcare: What is the evidence? How strong is the evidence? What is the value of the model/therapy/process? Can we validate the model? As the role of modeling in population health has become more widespread and increasingly complex, the tools and methodologies employed for model evaluation and validation must continue to keep pace (Fone et al 2003; Kopec et al 2010).

While the use of modeling in areas like comparative effectiveness analysis and infectious disease surveillance can ultimately trickle down, the individual clinician needs help with day-to-day and patient-topatient decision-making. If the aforementioned intensive care physician is faced with upwards of 200 variables on each day's rounds, it follows that an enormous of amount of data must exist for the population of patients treated in the ICU. How does the physician utilize this vast wealth of population data to inform the decision making process? A number of entities (e.g., Archimedes (Eddy 2006)) have seized upon the opportunity for data mining and model development employing these large sets of data. The re- 


\section{Fackler and Spaeder}

sult is population and physiology based models that provide a number of outputs (e.g. clinical outcomes, quality of life) for a variety of healthcare questions.

\section{REFERENCES}

Branson, R., J. Johannigman, R. Campbell and K. Davis. 2002. "Closed-loop mechanical ventilation." Respir Care, 47(4),427-51; discussion 451-3.

Burke, D., J. Epstein, D. Cummings, J. Parker, K. Cline, R. Singa and S. Chakravarty. 2006. "Individualbased computational modeling of smallpox epidemic control strategies." Acad Emerg Med, 13(11),1142-1149.

Chase, J., A. Le Compte, J. Presier, G. Shaw, S. Penning and T. Desaive. 2011. "Physiologic modelling, tight glycemic control, and the ICU clinician: what are the models and how can they affect practice?" Ann Intensive Care, 1(1), 11.

de Asla, R., Benis AM, Jurado RA, Litwak RS. 1984. Management of postcardiotomy hypertension by microcomputer-controlled administration of sodium nitroprusside. J Thorac Cardiovasc Surg. 89(1):115-120.

Eddy, D. 2006. "Linking electronic medical records to large-scale simulation models: can we put rapid learning on turbo?" Health Aff (Millwood), 26(2),125-36.

Eddy D., J. Adler and B. Patterson. 2011. Individualized guidelines: the potential for increasing quality and reducing costs. Ann Intern Med. 2011;154(9):627-634.

Fackler, J., C. Watts, A. Grome, T. Miller, T. Crandall and P. Pronovost. 2009. "Critical care physician cognitive task analysis: an exploratory study." Critical Care, 13:R33.

Fone, D., S. Hollinghurst, M. Temple, A. Round, N. Lester, A. Weightman, K. Roberts, E. Coyle, G. Bevan and S. Palmer. 2003. "Systematic review of the use and value of computer simulation modeling in population health and health care delivery." J Public Health Med, 25(4):325-335.

Fried, R., U. Gather, M. Imhoff. 2001. Online pattern recognition in intensive care medicine. Proc AMIA Symp, 184-188.

Imhoff, M. and R. Fried. 2002. "Detecting relationships between physiological variables using graphical modeling." In Proceedings of the AMIA Symposium, 340-344.

Kavanagh, B. and K. McCowen. 2010. "Glycemic control in the ICU." New England Journal of Medicine, 363, 2540-6.

Kiersey, D., A. Faulconer and R. Bickford. 1954. "Automatic electroencephalographic control of thiopental anesthesia." Anesthesiology,15(4), 356-364.

Krinsley, J. 2008. "Glycemic variability: a strong independent predictor of mortality in critically ill patients." Crit Care Med, 36(11), 3008-13.

Kopec, J., P. Fines, D. Manuel, D. Buckeridge, W. Flanagan, J. Oderkirk, Abrhamowicz, S. Harper, B. Sharif, A. Okhmatovskaia, E. Sayre, M. Rahman and M. Wolfson. 2010. "Validation of populationbased disease simulation models: a review of concepts and methods." BMC Public Health, 10, 710.

Miller, G. 1956. "The magical number seven plus or minus two: some limits on our capacity for processing information." Psychological Review, 63(2), 81-97.

Sieck, W., G. Klein, D. Peluso, J. Smith and D. Harris-Thompson. 2004. "A model of sensemaking." In U.S. Army Research Institute for Behavioral and Social Sciences A, VA. Fairborn, OH: Klein Associates.

Stahl, J. 2008 "Modelling methods for pharmacoeconomics and health technology assessment." Pharmacoeconomics, 26(2), 131-148.

\section{AUTHOR BIOGRAPHIES}

JAMES FACKLER is an Associate Professor in the Departments of Anesthesiology, Critical Care Medicine and Pediatrics at the Johns Hopkins University School of Medicine. He received his B.S. in biology 


\section{Fackler and Spaeder}

from the University of Illinois and his M.D. from Rush Medical College. He undertook his graduate medical training in Pediatrics, Anesthesiology and Pediatric Critical Care Medicine at the Johns Hopkins Hospital. His research interests are in the introduction and maintenance of knowledge based automation in the intensive care unit and pushing the walls of intensive care to include monitoring and assistance to chronically critically ill children at home

MICHAEL SPAEDER is an Assistant Professor in the Department of Pediatrics at the George Washington University School of Medicine and Health Sciences and an Attending Physician in the Division of Critical Care Medicine at the Children's National Medical Center. He received his B.S. in mathematics from Trinity College and his M.S. in mathematical statistics and M.D. from the George Washington University. He undertook his graduate medical training in Pediatrics and Pediatric Critical Care Medicine at Brown University/Hasbro Children's Hospital and the Johns Hopkins Hospital, respectively. Prior to his medical training, he was employed as a statistical consultant with the Economics Consulting and Quantitative Analysis group at Ernst \& Young, LLP in Washington, DC. His research interests include the predictive modeling of pediatric viral respiratory illness and outcomes analyses of children with severe viral respiratory illness. 